\title{
Effects of Yougui Pill Combined With Diacerein on Gonitis and Serum Levels of Toll-Like Receptor 4, Matrix Metalloproteinase-3 and Nitric Oxide
}

\author{
QIWEI MA, YAN ZHOU ${ }^{1}$, XUEJUAN YU ${ }^{2}, X$ IAOLI TANG ${ }^{3}$ AND CHENG ZANG ${ }^{1 *}$
}

Department of Orthopaedics, Zhuji Affiliated Hospital of Shaoxing University, Shaoxing 311800, ${ }^{1}$ Qingdao Hospital of Traditional Chinese Medicine (Qingdao Hiser Hospital), ²Qingdao Traffic Hospital, Qingdao 266000, 3Jingzhou Traditional Chinese Medicine Hospital, Hubei Province 430000, China

Ma et al.: Yougui Pill Effects Combined With Diacerein on Gonitis and Serum Levels

To observe the efficacy of Yougui pill combined with diacerein on gonitis and the effects of serum Tolllike receptor 4, matrix metalloproteinase-3 and nitric oxide. A total of 132 patients with gonitis treated in our hospital from January 2019 to October 2020 were selected and divided according to their treatment regimen. Efficacy: After $8 \mathrm{w}$ of treatment, 28 patients had complete remission in the observation group, accounting for $42.42 \%, 25$ patients had partial remission, accounting for $37.88 \%$ and 8 patients had remission, accounting for $12.12 \%$; in the control group, 14 patients had complete remission, accounting for $21.21 \%, 21$ patients had partial remission, accounting for $31.82 \%$ and 18 patients had remission, accounting for $27.27 \%$; in the observation group, the overall response rate was higher than that in the control group and it was statistically significant $(\mathrm{p}<\mathbf{0 . 0 5})$. Serum: After $8 \mathrm{w}$ of treatment, Toll-like receptor 4, matrix metalloproteinase-3 and nitric oxide, erythrocyte sedimentation rate and $\mathrm{C}$-reactive protein in both groups were largely decreased compared with those before treatment $(p<0.05)$ and after $8 \mathrm{w}$ of treatment in the observation group, Toll-like receptor 4, matrix metalloproteinase-3 and nitric oxide, erythrocyte sedimentation and $C$-reactive protein were lower compared with the control group ( $p<0.05)$. Bone and joint function: The pain, daily activities, stiffness and total scores in both groups after $8 \mathrm{w}$ of treatment were greatly decreased compared with those before treatment $(p<0.05)$ and the above scores were lower in the observation group after $8 \mathrm{w}$ of treatment compared with those in the control group $(p<0.05)$. Safety: In the observation group, gastric discomfort occurred in 2 patients, dry mouth in 3 patients and fatigue in 1 patient; in the control group, gastric discomfort occurred in 2 patients and dry mouth in 1 patient; the rate of adverse effects in the observation group was not statistically different from that in the control group ( $p>0.05)$. The combination of Yougui pill and diacerein improved the efficacy and decreased the expression of serum related factors without increasing adverse effects in patients with gonitis.

Key words: Yougui pill, diacerein, gonitis, Toll-like receptor 4, matrix metalloproteinase-3, nitric oxide

Gonitis is a common degenerative bone and joint disease in middle-aged and elderly people, which often involves bone, periosteum and periarticular supporting structures and clinically manifests with joint pain, swelling and limited function. Factors such as TollLike Receptor 4 (TLR-4), Matrix Metalloproteinase-3 (MMP-3) and Nitric Oxide (NO) play important roles in cartilage damage, periosteal proliferation and chronic inflammation in patients with gonitis ${ }^{[1]}$. At present, there is no radical treatment for this disease in western medicine clinic and most symptomatic treatments such as anti-inflammation and analgesia are used. Diacerein, an interleukin-1 inhibitor with pharmacological effects of inducing chondrogenesis, anti-inflammation, analgesia, antipyresis and so on, is one of the common drugs used in the current western medicine for the treatment of osteoarthritis. However, it can cause diarrhea, abdominal pain and other adverse effects, and the symptoms will still worsen after drug withdrawal ${ }^{[2]}$. Yougui pill has the efficacy of warming Yang and

*Address for correspondence

E-mail: Zangcheng1000@163.com 
invigorating the kidney and filling the spermatozoon for pulp supplementation, and is commonly used in clinical practice for low back and knee acid cooling caused by deficiency of kidney Yang ${ }^{[3]}$. In this study, we applied it to the treatment of gonitis to observe the effect of Yougui pill combined with diacerein on gonitis and the serum levels of TLR-4, MMP-3 and NO, which are reported as follows.

\section{MATERIALS AND METHODS}

\section{Inclusion and exclusion criteria:}

The inclusion criteria were Gonitis met the criteria of the "Guide for the Diagnosis and Treatment of Osteoarthritis (2018 Edition)"[4]; Age $\geq 18 \mathrm{y}, \leq 75 \mathrm{y}$; Syndrome differentiation met the criteria of the "Expert Consensus on Traditional Chinese Medicine Diagnosis and Treatment of Knee Osteoarthritis (2015 Edition)"[5]; All were primary gonitis and the clinical data were complete.

Exclusion criteria were patients with a history concerning intraarticular treatment or knee surgery; Patients with a history of relevant drug allergies; Patients with other serious somatic diseases; Patients with other bony diseases such as knee fracture, rheumatoid and gout; and patients who were pregnant or lactating women.

\section{General data:}

A total of 132 patients with gonitis treated in our hospital from January 2019 to October 2020 were selected and divided according to their treatment regimen and in the control group, 66 patients were given diacerein treatment, including 38 males and 28 females; age range of 36-70 y, mean $(56.36 \pm 8.09) \mathrm{y}$; disease duration of $0.5-15 \mathrm{y}$, mean $(7.96 \pm 2.15) \mathrm{y}$. In the observation group, 66 patients were given combined therapy with Yougui pill and diacerein, including 34 males and 32 females; age range of $35-70 \mathrm{y}$, mean $(57.12 \pm 8.52) \mathrm{y}$; disease duration of $0.5-15 \mathrm{y}$, mean $(7.89 \pm 2.27) \mathrm{y}$. The general data of patients in the two groups were compared and there was no statistical significance $(\mathrm{p}>0.05)$.

\section{Methods:}

The control group was treated with diacerein capsules (packaged by Kunming Jida Pharmaceutical Co., Ltd., specification: $50 \mathrm{mg}$, Saudi Food and Drug Authority (SFDA) Approval No. J20150097) orally $50 \mathrm{mg} /$ time for 2 times/d. The observation group was administrated with Yougui pill (Tongrentang Pharmaceutical Factory, Beijing Tongrentang Co., Ltd., specification: $9 \mathrm{~g} /$ pill, SFDA Approval No. Z11021040) combined with diacerein treatment and diacerein administration was the same as that in the control group, which was orally administrated with Yougui pill 1 pill/time, 3 times/d. Both groups were evaluated for efficacy after $8 \mathrm{w}$ of treatment.

\section{Observation indicators and detection methods:}

Efficacy criteria: They were formulated with reference to the "Guiding Principles for Clinical Investigation of New Chinese Medicines ${ }^{[6]}$ and Western Ontario and McMaster Universities Arthritis Index (WOMAC) Scores", Complete remission-After treatment, symptomatic signs such as joint pain, morning stiffness, joint swelling, local skin fever in the joints and unfavorable flexion and extension of the joints disappeared, the WOMAC integral decreased by $\geq 95$ $\%$ and the joint functional activity returned to normal; Partial remission-With treatment, the above symptom signs significantly relieved, $75 \% \leq$ WOMAC integral reduction $<95 \%$ and the joint functional activity basically returned to normal; Remission-With treatment, the above symptom signs relieved, $30 \% \leq$ WOMAC integral reduction $<75 \%$ and the joint functional activity significantly progressed; Ineffectiveness-Did not meet the above criteria.

Serum factors: Blood was taken before treatment and $8 \mathrm{w}$ after treatment to detect the indicators of TLR-4, MMP-3, NO, Erythrocyte Sedimentation (ESR) and C-Reactive Protein (CRP), venous blood was drawn from the upper limbs over $8 \mathrm{~h}$ of fasting, ESR was detected by the Westergren method and serum was collected after centrifugation to detect TLR-4, MMP3, NO and CRP by enzyme-linked immunosorbent assay. Instrument RT-96A microplate reader, Shenzhen Mairui Medical Electronic Co., Ltd., kit: Shanghai Mlbio Biotechnology Co., Ltd.

WOMAC score ${ }^{[7]}$ : This includes 3 major aspects of pain, daily activities, stiffness, the total scores were 20 , 8 and 68 points, respectively; the higher the score, the more severe the disease.

Safety evaluation: This includes gastric discomfort, dry mouth, fatigue and so on during medication.

\section{Statistical methods:}

The data were processed using Statistical Package for the Social Sciences (SPSS) 19.0 and the $(\overline{\mathrm{x}} \pm \mathrm{s})$ was applied to describe the measurement indexes and the t-test was applied for comparison, while the number of cases (percentage) was applied to describe the counting data and the $\chi^{2}$ test was applied for comparison and statistical significance was considered at $\mathrm{p}<0.05$. 


\section{RESULTS AND DISCUSSION}

The total effective rate in observation group was higher than that in control group and there was statistical significance $(\mathrm{p}<0.05)$. See Table 1 .

TLR-4, MMP-3 and NO were greatly decreased in both groups after treatment $(\mathrm{p}<0.05)$, and the levels of TLR4, MMP-3 and NO were lower in the observation group than those in the control group after treatment $(\mathrm{p}<0.05)$. See Table 2.

ESR and CRP in both groups decreased greatly after treatment $(\mathrm{p}<0.05)$, and ESR and CRP in the observation group were lower after treatment compared with those in the control group $(\mathrm{p}<0.05)$. See Table 3.

The pain, daily activities, stiffness and total scores of the two groups largely decreased after treatment $(p<0.05)$ and the above scores of the observation group were lower than those of the control group after treatment $(p<0.05)$. See Table 4.

The rate of adverse effects in the observation group was not statistically different from that in the control group ( $>>0.05)$. See Table 5.

TABLE 1: COMPARISON OF EFFICACY BETWEEN TWO GROUPS [n (\%)]

\begin{tabular}{|c|c|c|c|c|c|c|}
\hline Group & $\begin{array}{c}\text { Number of } \\
\text { cases }\end{array}$ & Full mitigation & $\begin{array}{c}\text { Partial } \\
\text { mitigation }\end{array}$ & Mitigation & Invalid & Total efficiency \\
\hline Control group & 66 & $14(21.21)$ & $21(31.82)$ & $18(27.27)$ & $13(19.70)$ & $53(80.30)$ \\
\hline $\begin{array}{l}\text { Observation } \\
\text { Group }\end{array}$ & 66 & $28(42.42)$ & $25(37.88)$ & $8(12.12)$ & $5(7.58)$ & $61(92.42)$ \\
\hline$\chi^{2}$ & & & & & & 4.117 \\
\hline $\mathrm{p}$ & & & & & & 0.043 \\
\hline
\end{tabular}

TABLE 2: COMPARISON OF SERUM TLR-4, MMP-3 AND NO LEVELS BETWEEN THE TWO GROUPS ( $\overline{\mathrm{x}} \pm \mathrm{s})$

\begin{tabular}{lccccccc}
\hline \multirow{2}{*}{ Group } & $\begin{array}{c}\text { Number of } \\
\text { cases }\end{array}$ & $\begin{array}{c}\text { Before } \\
\text { treatment }\end{array}$ & $\begin{array}{c}\text { After } \\
\text { treatment }\end{array}$ & $\begin{array}{c}\text { Before } \\
\text { treatment }\end{array}$ & $\begin{array}{c}\text { After } \\
\text { treatment }\end{array}$ & $\begin{array}{c}\text { Before } \\
\text { treatment }\end{array}$ & $\begin{array}{c}\text { After } \\
\text { treatment }\end{array}$ \\
\cline { 3 - 7 } $\begin{array}{l}\text { Control group } \\
\text { Observation }\end{array}$ & 66 & $18.96 \pm 5.14$ & $15.26 \pm 3.01^{*}$ & $25.23 \pm 5.14$ & $19.85 \pm 3.23^{*}$ & $58.56 \pm 15.36$ & $50.23 \pm 11.47^{*}$ \\
Group & 66 & $19.04 \pm 4.86$ & $11.47 \pm 2.52^{*}$ & $24.98 \pm 4.76$ & $13.05 \pm 2.94^{*}$ & $60.04 \pm 14.27$ & $40.25 \pm 8.14^{*}$ \\
$t$ & & 0.092 & 7.843 & 0.290 & 12.648 & 0.573 & 5.765 \\
p & & 0.927 & 0.000 & 0.772 & 0.000 & 0.567 & 0.000 \\
\hline
\end{tabular}

Note: Compared with this group before treatment, ${ }^{*} p<0.05$

TABLE 3: COMPARISON OF ESR, CRP BETWEEN TWO GROUPS ( $\bar{x} \pm s)$

\begin{tabular}{|c|c|c|c|c|c|}
\hline \multirow{2}{*}{ Group } & \multirow{2}{*}{ Number of cases } & \multicolumn{2}{|c|}{$\operatorname{ESR}(\mathrm{mm} / \mathrm{h})$} & \multicolumn{2}{|c|}{ CRP (mg/l) } \\
\hline & & Before treatment & After treatment & Before treatment & After treatment \\
\hline Control group & 66 & $18.45 \pm 3.63$ & $12.05 \pm 1.98^{*}$ & $7.05 \pm 1.23$ & $4.25 \pm 0.98^{*}$ \\
\hline Observation group & 66 & $18.51 \pm 3.27$ & $9.58 \pm 1.24^{*}$ & $7.14 \pm 1.06$ & $2.89 \pm 0.74^{*}$ \\
\hline $\mathrm{t}$ & & 0.100 & 8.589 & 0.450 & 8.997 \\
\hline $\mathrm{p}$ & & 0.921 & 0.000 & 0.653 & 0.000 \\
\hline
\end{tabular}

Note: Compared with this group before treatment, * $p<0.05$

TABLE 4: COMPARISON OF WOMAC SCORES BETWEEN GROUPS ( $\overline{\mathbf{x}} \pm \mathbf{s})$

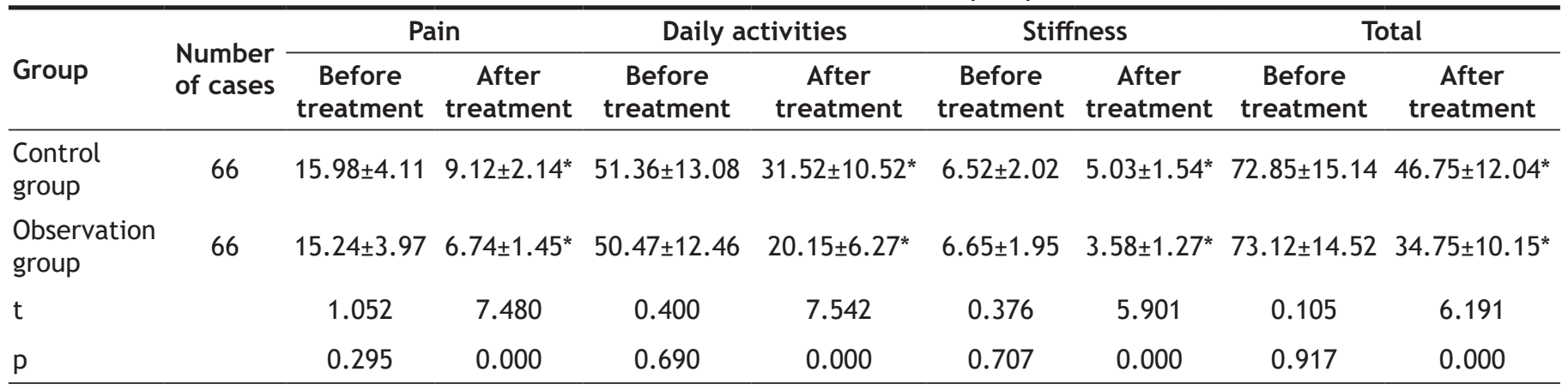

Note: Compared with this group before treatment, ${ }^{*} \mathrm{p}<0.05$ 
TABLE 5: TWO SETS OF SAFETY EVALUATIONS [n (\%)]

\begin{tabular}{|c|c|c|c|c|c|}
\hline Group & Number of cases & $\begin{array}{c}\text { Stomach } \\
\text { discomfort }\end{array}$ & Dry mouth & Fatigue & Total \\
\hline Control group & 66 & $2(3.03)$ & $1(1.52)$ & $0(0.00)$ & $3(4.55)$ \\
\hline Observation group & 66 & $2(3.03)$ & $3(4.55)$ & $1(1.52)$ & $6(9.09)$ \\
\hline$\chi^{2}$ & & & & & 1.073 \\
\hline$p$ & & & & & 0.300 \\
\hline
\end{tabular}

Gonitis is a common clinical degenerative change of the lower extremities that occurs mostly in the middleaged and elderly, is more common in the $50-60$ y age group and is more common in women than men, which is associated with disturbed sex hormone levels and exacerbated bone loss after women enter menopause ${ }^{[8]}$. The incidence of gonitis is increasing with the aging socio population in China ${ }^{[9]}$. Since cartilage damage, periosteal hyperplasia and chronic inflammation are the main causes of knee pain and dysfunction, the current treatment of gonitis in western medicine is based on symptomatic treatment such as resting, physiotherapy, anti-inflammation, analgesia, nutritious cartilage and lubricating joints, which can relieve the symptoms to a certain extent. However, some patients still have suboptimal efficacy and patients with severe disease need to accept knee replacement therapy ${ }^{[10]}$.

Diacerein is currently a common drug used in clinical treatment of gonitis and its active ingredient is diacetylrhein, which belongs to the anthraquinone class of compounds and reduces prostaglandin synthesis by inhibiting interleukin-1. Besides anti-inflammatory and analgesic effects, it can also induce chondrogenesis to promote cartilage repair ${ }^{[11]}$. Louthrinoo et al. ${ }^{[12]}$ found that diacerein treatment had satisfactory efficacy in rheumatoid arthritis with poor response to methotrexate. Wang et al..$^{[13]}$ used celecoxib combined with diacerein to treat elderly degenerative knee osteoarthritis with satisfactory efficacy, producing complementary effects and the effect was more sustained and safe.

The occurrence of gonitis is closely related to chronic exertion, poor long-term posture, weight bearing, obesity and cold affected Bone Mineral Density (BMD). Yougui pill is an essential medicine for warming the kidney and strengthening Yang in traditional Chinese medicine and belongs to the tonic class, which has the effects of warming the kidney to dissipate cold and strengthening the tendons and bone. Modern research has found its anti-inflammatory, analgesic, osteoporotic and microcirculation improving and pharmacological effects, and it is clinically used to treat hyposexual function of kidney yang deficiency, osteoporosis, chronic bronchitis, persistent asthma, sciatica, progressive muscular dystrophy, hereditary cerebellar ataxia, breast cysts, leukopenia, lupus erythematosus, chronic diarrhea, chronic gastritis, gastric ulcer, major bleeding, after induced abortion, psoas muscle strain, unexplained postpartum fever, chronic renal failure, nephrotic syndrome and other diseases ${ }^{[14]}$. Shao et $a l .{ }^{[15]}$ found that the treatment of Yang deficiency cold coagulation type knee osteoarthritis by Yougui pill combined with ginger moxibustion has a significant effect, which can reduce pain and inflammatory symptoms, improve immunity and have a high safety.

In this study, we referred to the "Guiding Principles of Clinical Investigation of New Chinese Medicines" and WOMAC score to evaluate the efficacy and found that the combination of Yougui pill and diacerein improved the efficacy and decreased the pain, daily activity, stiffness scores and WOMAC total scores in patients with gonitis and it had a good safety profile, without increasing the rate of adverse reactions such as stomach discomfort, dry mouth and tongue, and fatigue. This is due to the fact that aconitine type alkaloids contained in aconite in the Yougui pill can exert antiinflammatory and analgesic effects by exciting the pituitary adrenocortical system ${ }^{[16]}$. Staghorn gum extract can promote lymphocyte transformation and upregulate articular chondrocyte kinase expression to repair damaged cartilage and has anti-inflammatory and analgesic effects, and its glycine contained can also promote calcium ion absorption ${ }^{[17]}$. Radix Rehmanniae Praeparata extract enhances bone marrow hematopoiesis, modulates immunity, antioxidation and anti-inflammation ${ }^{[18]}$. Angelica sinensis polysaccharides have immunomodulatory effects and its volatile oil has anti-inflammatory, microcirculation improving and other pharmacological effects ${ }^{[19]}$.

TLR4 is involved in regulating innate immune response, inflammatory response and arthrohydrops of gonitis patients causes synovial inflammation and joint structure damage through TLR4 pathway ${ }^{[20]}$. MMP-3 can act on the extracellular matrix of articular cartilage to degrade proteoglycans and collagens in it 
to destroy articular cartilage structure, which cannot activate interstitial collagenases and destroy cartilage structure $^{[21]}$. NO functions as both a second messenger and neurotransmitter, and interleukin-1 can induce chondrocytes to synthesize NO and play an important role in the inflammatory reaction ${ }^{[22]}$. ESR and CRP are commonly used inflammatory indicators, which can sensitively reflect the body's inflammatory status ${ }^{[23]}$. In this study, by detecting serum TLR-4, MMP-3, NO, CRP and ESR, we found that the combination of Yougui pill and diacerein decreased the expression of cartilage damage related factors in serum and alleviated the body's immune inflammatory response. This is one of the important mechanisms for its treatment of gonitis.

In conclusion, the combination of Yougui pill and diacerein improved the efficacy and decreased the expression of serum related factors without increasing adverse effects in patients with gonitis.

\section{Authors' contributions:}

Qiwei Ma and Xiaoli Tang have contributed equally to this work.

\section{Conflict of interests:}

The authors declared no conflicts of interest.

\section{REFERENCES}

1. Ko JH, Kang YM, Yang JH, Kim JS, Lee WJ, Kim SH, et al. Regulation of MMP and TIMP expression in synovial fibroblasts from knee osteoarthritis with flexion contracture using adenovirus-mediated relaxin gene therapy. Knee 2019;26(2):317-29.

2. Siddiqui B, Rehman AU, Haq IU, Ahmad NM, Ahmed N. Development, optimisation and evaluation of nanoencapsulated diacerein emulgel for potential use in osteoarthritis. J Microencapsul 2020;37(8):595-608.

3. Chunlu Y, Fangyu A, Yongfeng W. Effects of Yougui pill on the expression of genes regulated by apoptosis and matrix degradation in murine cartilage tissue in a knee osteoarthritis model. Chin J Osteoporos Bone Miner Salt Dis 2020;13(1):418.

4. Joint Surgery Group, Chinese Society of Osteology, Chinese Medical Association. Diagnosis and treatment guidelines for osteoarthritis (2018 Edition). Chin J Orthop 2018;38(12):70515.

5. Orthopedics Professional Committee of China Association for the Promotion of Traditional Chinese Medicine, Joint Working Committee of Orthopedics and Traumatology Professional Committee of Chinese Academy of Integrated Traditional and Western Medicine. Expert consensus on TCM diagnosis and treatment of knee osteoarthritis (2015 Edition). J Tradit Chin Orthop Traumatol 2015;27(7):4-5.

6. Xiaoyu Z. Guiding principles for clinical research of new chinese medicines]. Beijing: China Pharmaceutical Science and Technology Press 2002:67-8.
7. Bellamy N. WOMAC: a 20-year experiential review of a patient-centered self-reported health status questionnaire. J Rheumatol 2002;29(12):2473-6.

8. Nishitani K, Kuriyama S, Nakamura S, Morita Y, Ito H, Matsuda S. Valgus position of the femoral component causes abnormal kinematics in the presence of medial looseness in total knee arthroplasty: A computer simulation model of TKA for valgus knee osteoarthritis. Knee Surg Sports Traumatol Arthrosc 2019;27(7):2051-60.

9. O'Brien AT, El-Hagrassy MM, Rafferty H, Sanchez P, Huerta $\mathrm{R}$, Chaudhari $\mathrm{S}$, et al. Impact of therapeutic interventions on pain intensity and endogenous pain modulation in knee osteoarthritis: A systematic review and meta-analysis. Pain Med 2019;20(5):1000-11.

10. Bernardo-Bueno MM, Gonzalez-Suarez CB, Malvar AK, Cua $\mathrm{R}$, Feliciano D, Tan-Sales BG, et al. Stratifying minimal versus severe pain in knee osteoarthritis using a musculoskeletal ultrasound protocol. J Ultrasound Med 2019;38(6):1411-23.

11. Aziz DE, Abdelbary AA, Elassasy AI. Investigating superiority of novel bilosomes over niosomes in the transdermal delivery of diacerein: in vitro characterization, ex vivo permeation and in vivo skin deposition study. J Liposome Res 2019;29(1):7385.

12. Louthrenoo W, Nilganuwong S, Nanagara R, Siripaitoon B, Basset SC. Diacerein for the treatment of rheumatoid arthritis in patients with inadequate response to methotrexate: A pilot randomized, double-blind, placebo-controlled add-on trial. Clin Rheumatol 2019;38(9):2461-71.

13. Guodong W, Lingyun Y, Bin H. Efficacy and safety of celecoxib combined with diacerein for the treatment of elderly patients with degenerative knee osteoarthritis: protocol for a randomized controlled clinical trial. J Clin Rehab Tissue Eng Res 2017;36(21):5747-51.

14. Chunlu Y, Fangyu A, Yongqi L. Mechanism of regulation of Yougui Pill via IL-6/STAT3 signaling pathway on cartilage tissue degeneration in a knee osteoarthritis model rat. Chin J Exp Formula 2020;26(1):17-23.

15. Jieqi S, Zhijiang Z, Min Y. Clinical effects of Yougui pill combined with moxibustion of ginger for treatment of Yang deficiency cold coagulation type of knee osteoarthritis. Chin Med Herald 2018;15(10):106-9.

16. Yanqing Z, Sai L, Lan P. Probing the mechanism of action of monkshood warming Yang based on network pharmacology. J Beijing Univ Tradit Chin Med 2019;42(2):143-8.

17. Min L, Xiaolin S, Xu Chao. Traditional Chinese medicine compounds and target network pharmacology of Yougui pill for anti-osteoporosis. Chin J Orthop Traumatol 2020;33(10):9337.

18. Peng F, Donghao G, Weiguo W. Molecular mechanism study of Radix Rehmanniae Praeparata intervention for osteoporosis based on network pharmacology. Shandong J Tradit Chin Med 2020;39(2):177-84.

19. Peiliang D, Hui L, Hua H. A review of Angelica sinensis and its drug pairs. Inform Tradit Chin Med 2019;36(2):127-30.

20. Huang X, Qiao F, Xue P. The protective role of microRNA$140-5 p$ in synovial injury of rats with knee osteoarthritis via

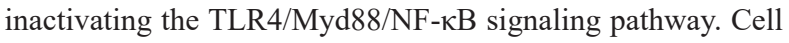
Cycle 2019;18(18):2344-58.

21. Georgiev T, Ivanova M, Kopchev A, Velikova T, Miloshov A, Kurteva E, et al. Cartilage oligomeric protein, matrix metalloproteinase-3 and Coll2-1 as serum biomarkers in knee osteoarthritis: A cross-sectional study. Rheumatol Int 2018;38(5):821-30. 
22. Hoffmeister E. Study finds no association between osteoarthritis therapy and occurrence of knee replacement. Lippincotts Bone Joint Newslett 2019;25(1):1-5.

23. Abou-Raya SN, Meyers DE, Sayed EA, Kamal-El Deen MA. Study of metabolic syndrome frequency in elderly patients with knee osteoarthritis and its impact on the physical activity. Egyptian J Obes Diabetes Endocrinol 2018;4(3):73-81.
This is an open access article distributed under the terms of the Creative Commons Attribution-NonCommercial-ShareAlike 3.0 License, which allows others to remix, tweak, and build upon the work non-commercially, as long as the author is credited and the new creations are licensed under the identical terms

This article was originally published in a special issue, "Novel Therapeutic Approaches in Biomedicine and Pharmaceutical

Sciences" Indian J Pharm Sci 2021:83(6) Spl Issue "118-123" 